\title{
Curso de dissecação inguinal em cadáveres - um relato de experiência
}

\author{
Course of inguinal dissection in corpses - an experience report \\ Curso de disección inguinal en cadáveres - un informe de experiencia
}

Matheus Albert de Souza Puerro ${ }^{1 *}$, Aysha Nayane Lisboa Franco ${ }^{1}$, Diego Teles Borges Leal ${ }^{1}$, Gabriela de Arruda Custodio ${ }^{1}$, Guilherme Alves da Silva ${ }^{1}$, Juliana Miranda Benício ${ }^{1}$, Larisse Lima da Silva Nascimento ${ }^{1}$, Renan Kleber Costa Teixeira ${ }^{1}$, Franklin Coelho Nascimento ${ }^{1}$.

\section{RESUMO}

Objetivo: Este estudo tem como objetivo descrever a experiência de alunos graduandos de Medicina, discentes do Centro Universitário Metropolitano da Amazônia, exemplificando como a dissecação contribui para a formação de habilidades manuais e intelectuais destes estudantes e assim cumprindo as competências instituídas pelas Diretrizes Curriculares Nacionais do Curso de Medicina. Relato de experiência: Trata-se de um estudo descritivo, do tipo relato de experiência, a partir de um evento, que teve duração de cinco dias. $O$ curso foi iniciado com uma atividade teórica, na qual os alunos puderam aprender mais sobre os instrumentais cirúrgicos, que seriam utilizados durante a dissecação. Além disso, foi ministrada uma aula de revisão, a respeito da anatomia da região inguinal. Seguiu-se um treinamento prático, sendo realizado em simuladores antes da dissecação no cadáver, com a finalidade de aprimorar as habilidades manuais dos discentes. Considerações finais: $O$ curso de dissecção proporcionou a possibilidade de vivenciar as bases do eixo morfofuncional em sua plenitude, percebendo a importância das relações anatômica-patológica.

Palavras-chave: Dissecação, Anatomia, Cadáver, Ensino.

\section{ABSTRACT}

Objective: This study has the goal to describe the experience of medical undergraduates, students from Centro Universitário Metropolitano da Amazônia, exemplifying how the dissection contribute to the formation of manual and intellectual abilities of those students, accomplishing the competences instituted by the national curriculum guidelines from the medicine course. Experience report: This is a descriptive study, an experience report, based on an event, which lasted five days. The course started with a theoretical activity, in which students could learn more about surgical instruments, which would be used during dissection. In addition, a review class was given, regarding the anatomy of the inguinal region. A practical training followed, being carried out in simulators before dissection in the corpse, in order to improve the students' manual skills. Final considerations: the dissection course provided the possibility to experiment the morphofunctional axis bases in it's plenitude, realizing the importance of the relation between anatomy and pathology.

Key words: Dissection, Anatomy, Corpse, Education.

\section{RESUMEN}

Objetivo: El objetivo de este estudio es describir la experiencia de estudiantes de medicina de pregrado, estudiantes del Centro Universitario Metropolitano de Amazonia, ejemplificando cómo la disección contribuye a la formación de habilidades manuales e intelectuales de estos estudiantes y cumpliendo así las competencias establecidas por las Pautas Nacionales del Plan de Estudios de Medicina. Informe de experiencia: Es un estudio descriptivo, un informe de experiencia, basado en un evento, que duró cinco días. El curso comenzó con una actividad teórica, en la que los estudiantes podían aprender más sobre los

\footnotetext{
${ }^{1}$ Centro Universitário Metropolitano da Amazônia - UNIFAMAZ, Belém - PA. *E-mail: albertpuerro@gmail.com
} 
instrumentos quirúrgicos, que se utilizarían durante la disección. Además, se impartió una clase de revisión sobre la anatomía de la región inguinal. Siguió una capacitación práctica, que se llevó a cabo en simuladores antes de la disección en el cadáver, con el fin de mejorar las habilidades manuales de los estudiantes. Consideraciones finales: El curso de disección proporcionó la posibilidad de experimentar las bases del eje morfofuncional en su plenitud, dándose cuenta de la importancia de las relaciones anatómico-patológicas.

Palabras clave: Disección, Anatomía, Cadáver, Enseñanza.

\section{INTRODUÇÃO}

A dissecação é uma prática de ensino usada ao longo de muitos séculos. Esta consiste na exploração das estruturas anatômicas de forma sistemática que ajuda a expor topograficamente os órgãos e tecidos para o ensino visual destes (NOBESCHI L, et al., 2018). O estudo anatômico é uma das mais antigas ciências médicas que constituem o cotidiano do estudante de medicina (LOPES ISL, et al., 2017). Este é de extrema importância devido a oportunidade que disponibiliza ao discente a desenvolver as habilidades e competências exigidas na atuação profissional cotidiana com mais segurança e pratica medica, por meio da dissecação. Porem este treinamento em peças anatômicas disponíveis para o ensino está se tornando uma pratica incomum (MEDEIROS ARC, et al., 2013; ÖZCAN S, et al., 2015)

O surgimento das novas tecnologias digitais ampliou o ensino da anatomia, possibilitando a substituição da dissecação por recursos de projeções audiovisuais e utilização de softwares (NOBESCHI L, et al., 2018; LOPES ISL, et al., 2017). Entretanto, o ensino baseado em cadáveres é reconhecido pelos alunos, docentes e clínicos como o melhor método para aprender anatomia, pois este inclui detalhes e variações anatômicas que são essenciais para a formação acadêmica de discentes da área da saúde, especialmente estudantes de medicina (LOPES ISL, et al., 2017; PATEL, K, et al., 2008; SHEIKH AH, et al., 2016). Ademais, muitos dos recursos tecnológicos de aprendizagem anteriormente citados, muitas vezes não poderão ser disponibilizados em muitas escolas de países em desenvolvimento, devido limitações financeiras (NOBESCHI L, et al., 2018).

A dissecção cadavérica é usada como uma ferramenta importante na aprendizagem da anatomia, devido à sua percepção efetividade para entender a classificação e inter-relacionamento de diferentes partes do corpo, integração da teoria e prática da anatomia, percepção mediada pelo toque, tridimensional perspectiva de estruturas e aplicação de habilidades práticas (ÖZCAN S, et al., 2015).

A nova diretriz curricular nacional do curso de graduação de medicina institui habilidades e competências relacionadas a liderança, tomada de decisão e etc (BRASIL., 2014). O currículo PBL centrado no aluno, também exige estas habilidades, incluindo a observação e interpretação, que são essenciais para a prática clínica eficaz (VAN WYKJ, RENNIE CO, 2015). Estas atribuições podem ser consolidadas a partir da dissecação ativa pelos estudantes, servindo como uma ferramenta para aquisição mais consolidada do conhecimento (MEDEIROS ARC, et al., 2013).

Os estudantes possuem forte opiniões sobre seus conhecimentos anatômicos (VAN WYKJ, RENNIE CO, 2015). Estes, sentem que seus conhecimentos de anatomia "não são bons o suficiente" para realizar as práticas médicas futuramente (NOBESCHI L, et al., 2018). Estas opiniões possui um efeito de longo alcance no desenvolvimento do currículo médico, afetando a aprendizagem crítica da anatomia (VAN WYKJ, RENNIE CO., 2015). Pois a entidade médica afirma que a anatomia permanece como um dos pilares da educação médica, por isso esta é introduzida no ensino logo no início do curso de medicina fornecendo bases para a linguagem médica, treinamento e prática (SINGH R, et al., 2015).

A aquisição do conhecimento anatômico por meio da dissecação possibilita o progresso científico e o desenvolvimento de capacitações como o trabalho em equipe, liderança, interação professor-aluno, aprendizado autodidata entre outros (MEDEIROS ARC, et al., 2013). Pois, esta é dotada de realismo e humanidade. Apesar de exigir maior tempo e competências, a dissecação, permite aprimorar a capacidade de observação, destreza manual, além de confrontar dilemas éticos-sociais que exigem uma postura responsável do discente de medicina (PONTINHA CM, SOEIRO C, 2014). 
A julgar pela importância da dissecação no processo de ensino-aprendizagem do estudante de medicina, justifica-se a realização deste relato de experiência, devido a escassa existência de trabalhos que avaliam a eficácia e a beneficias que este método suscita. Considera-se que este estudo contribuirá para o interesse das escolas médicas em conservar esta ferramenta de estudo, além de colaborar para a oferta de estudos nesta área.

Este estudo tem como objetivo descrever a experiência de alunos graduandos de Medicina, discentes do Centro Universitário Metropolitano da Amazônia, exemplificando como a dissecação contribui para a formação de habilidades manuais e intelectuais destes estudantes e assim cumprindo as competências instituídas pelas Diretrizes Curriculares Nacionais do Curso de Medicina.

\section{RELATO DE EXPERIÊNCIA}

O curso de dissecção é uma estratégia de treinamento de habilidades medicas do curso de medicina do Centro Universitário Metropolitano da Amazônia que ocorre semestralmente; em cada modulo é determinado um sitio topográfico para a realização das dissecções. Nesse relato será discutido sobre o modulo da dissecção da região inguinal e canal inguinal que ocorreu em dois cadáveres do sexo masculino. Nesse modelo de curso, discentes de medicina do $2^{\circ}$ ao $4^{\circ}$ semestre se inscrevem como monitores e são treinados previamente pelos professores organizadores. Durante os treinamentos, os monitores revisam e aprendem técnicas de didática, materiais cirúrgicos e anatomia sistêmica e topográfica da região a ser dissecada. Além disso, são padronizados os treinamentos realizados em simuladores de baixo custo.

As inscrições dos alunos no evento ocorreram por livre demanda com um número máximo de 10 inscritos. O período de inscrição início 10 dias antes do curso, sendo que após 2 horas do início da matriculas, todas as vagas foram preenchidas e já havia um cadastro de reserva de mais dez discentes. $O$ evento teve duração de cinco dias (carga horaria total de 20 horas) e ocorreu no final do período acadêmico dos discentes fora do horário das atividades curriculares. As atividades eram sempre iniciadas com uma breve atividade teórica, seguida com um treinamento prático. Foi realizado treinamento em simuladores no primeiro, segundo e quarto dia e no terceiro e quinto dia foi realizado a dissecção no cadáver.

No primeiro dia de treinamento, iniciou-se com a aula inaugural, onde foi apresentado o evento, informando os objetivos e a justificativa deste; bem como foi abordado às dinâmicas que seriam realizadas através da explanação do roteiro de prática (que também servia como uma fonte para orientar o estudo em casa), além de apresentar os monitores e professores participantes. Seguiu-se com uma palestra sobre os instrumentais cirúrgicos, ministrada por um dos monitores. Nessa foi abordado os instrumentais cirúrgicos básicos mais utilizados, além dos que seriam utilizados. Foi explicada a correta empunhadura, técnica de utilização e tempo principal. A seguir realizou-se uma atividade pratica onde foi simulando a arrumação de uma mesa cirúrgica, sendo reforçados os conceitos abordados na atividade teórica.

No segundo dia do curso, foi realizado um treinamento de incisão e dissecção, utilizando um modelo vegetal - uma laranja. A simulação da habilidade era realizada em duas fases, na primeira era realizado 0 treinamento de uma incisão vertical sob a casa tentando evitar lesionar os gomos da fruta. Após esse treinamento era realizado simulado a divulsão nas mesmas frutas onde se objetivava a separação da casca sem lesionar os gomos e após isolar os gomos sem danificá-los. Neste modelo era revisado os conceitos básicos de incisão e divulsão que serão utilizados na pratica de dissecção bem como era realizado uma revisão dos instrumentais utilizados.

O terceiro dia iniciou-se com uma breve revisão das estruturas a serem dissecadas com ênfase no canal inguinal e funículo espermático masculino visto sua maior importância anatômica e pelo fato de ambos os cadáveres serem do sexo masculino. Foi levemente abordado na aula teórica a correlação clínica-anatômica de algumas patologias, como hérnias de parede e varicocele. Essa palestra foi ministrada por um dos professores participantes do evento. Logo após iniciou-se a atividade de dissecção dos cadáveres sempre sob supervisão e com equipamentos de proteção individuais. Neste dia foram dissecados dois canais inguinais de um dos cadáveres. 
No quarto encontro, foi desenvolvido uma simulação que consistia em separar a parte muscular do tecido gorduroso de boi, por meio de divulsão. Para isso, os alunos foram subdivididos em duplas; e cada equipe tinha a disposição todos os instrumentais necessários para realizar a divulsão. O auxiliar fixava as estruturas, facilitando o procedimento para o outro aluno que estava divulsionando, e após um certo tempo, as duplas reversavam. O objetivo principal foi o aprimoramento das técnicas de divulsão e dissecação.

Após esta simulação, foi realizado um treinamento de nós e suturas. Para isso foi utilizado dois modelos de treinamento: 1) Uma esponja de cozinha revestida com Espuma Vinílica Acetinada (EVA) e fixada a uma prancha rígida para o treinamento de sutura; 2) Uma sonda vesical de demora posicionada dentro de uma esponja, onde foi realizado o treinamento dos nos manuais, por meio da confecção do nó de bailarina. Os discentes realizaram os treinamentos sob supervisão e orientação dos monitores e no caso de eventuais dúvidas com os professores presentes.

No último dia de curso, foi realizado a dissecção do funículo espermático e canal inguinal do segundo cadáver revisando a anatomia previamente identificada no primeiro. Ao termino da dissecção foi realizado uma breve revisão anatômica. Após foi realizado a síntese da pele do cadáver, revisando as técnicas apresentadas no dia anterior. Após os cadáveres foram armazenados de forma apropriada.

Figura 1 - Dissecação Canal Inguinal Direito



Fonte: Puerro MAS, et al., 2019

\section{DISCUSSÃO}

A Anatomia é uma disciplina curricular, cujo objetivo é levar o aluno de medicina, e de outros cursos da área da saúde, a aprender sobre estrutura, a função e a topografia dos órgãos que compõem os sistemas do corpo humano. Esse objetivo pode ser atingido de diversas formas tem sido atingido, desde uma completa ausência de um corpo para dissecação, com base em considerações teológicas e filosóficas, a uma observação passiva das dissecações realizadas pelos cirurgiões-barbeiros, até a reforma curricular que aconteceu nos Estados Unidos da América, no século XIX, que deu continuidade à tradição da dissecação, que existe desde a Grécia Antiga, como o ponto central do seu ensino (NOBESCHI L, et al., 2018).

Aprender usando dissecação de cadáveres possui inúmeras vantagens tal como; o aprimoramento a aprendizagem ativa e profunda, que fornece um preparo aos alunos para a prática clínica, prática de habilidades manuais e para entender o relacionamento entre os sintomas dos pacientes e patologia. Além 
disso, auxilia no desenvolvimento do profissionalismo médico, incluindo a competência de trabalho em equipe (ESTAI M, BUNT S, 2016).

Em analogia com o lluminismo, a metodologia ativa de ensino nas ciências médicas baseia-se na premissa de que todo aluno pode buscar e adquirir, as habilidades e o conhecimento para se tornar um profissional de excelência. Sendo que este é iluminado pela figura do professor, preceptor ou orientador, que representa as luzes da razão, já que possui o conhecimento consolidado (PONTINHA CM, SOEIRO C, 2014).

Nos últimos anos, em face do grande desenvolvimento da Biologia Celular, da Bioquímica e da Genética, algumas escolas médicas têm tomado caminhos, no que diz respeito ao ensino de anatomia, que poderão conduzir ao abandono da dissecação, já que esta exige tempo e investimento econômico das instituições, pois prática em cadáveres de seres humanos e em animais vivos está associada a custos elevados e por necessitar de instalações especializadas, além dos riscos de possíveis infecções durante a pratica (ESTAI M, BUNT S, 2016; RAMOS RFM, et al., 2016). Tal procedimento pode proporcionar reflexos negativos, levando a um ensino da Anatomia Humana que estará abaixo daquilo que se considera seguro e adequado para futuros médicos (PONTINHA CM, SOEIRO C, 2014).

Recursos computadorizados não são o suficiente para substituir os métodos tradicionais de ensino, envolvendo o uso de cadáveres e a dissecação dos mesmos. Porém, a associação correta das novas tecnologias como complementação dos métodos tradicionais, traz melhores resultados no aprendizado da anatomia humana, proporcionando um conhecimento mais amplo e seguro do aluno sobre anatomia (NOBESCHI L, et al., 2018).

Apesar de ser uma metodologia de ensino que exige tempo e grande consumidora de recursos econômicos, a dissecação do cadáver permite que o discente da área de saúde desenvolva a sua capacidade de observação e de destreza manual (LEMPP HK, 2005). A capacidade de observação e a destreza manual constituem umas das principais habilidades que um futuro médico precisa desenvolver, pois é por meio da primeira que o raciocínio clínico se torna possível. E com a segunda habilidade atende-se a necessidade de um procedimento cirúrgico, que por ventura venha a surgir.

O curso de dissecação anatômica realizado ofereceu o ambiente necessário e adequado, para a obtenção das habilidades supracitadas. Além disso, é oportuno frisar que os alunos que participaram do curso não são os únicos beneficiados. Pois, as estruturas do cadáver que antes estavam veladas puderam ser expostas para que outros discentes possam observar e complementar o seu próprio aprendizado. Já que, além de uma visão tridimensional, a dissecação anatômica proporciona a visualização de uma gama de variações anatômicas presentes em cadáveres, ao contrário do que eventualmente possa estar descrito nos livros (NOBESCHI L, et al., 2018).

\section{CONSIDERAÇÕES FINAIS}

As experiências desenvolvidas e adquiridas no curso de dissecção proporcionam a possibilidade de vivenciar as bases do eixo morfofuncional em sua plenitude, percebendo a importância das relações anatômicas com a fisiologia e histologia bem como para o desenvolvimento de patologias. Além disso, o curso proporcionou aos discentes habilidades (dissecção, manuseio de instrumentais), competências (ética, respeito e trabalho em grupo) e conhecimentos de imensa valia para a futura prática clínica e acadêmica.

\section{REFERÊNCIAS}

1. BRASIL. Ministério da Educação. Resolução nํ. 3, de 20 de junho de 2014. Diretrizes curriculares nacionais do curso de graduação em medicina. Diário Oficial da União, Brasília, DF, 2014.

2. ESTAI M, BUNT S. Best teaching practices in anatomy education: A critical review. Annals of Anatomy, 2016; 208: 151-157.

3. Perceptions of dissection by students in one medical school: beyond learning about anatomy. A qualitative study. Medical Education, 2005; 39(3): 318-25. 
4. LOPES ISL, et al. Use of human cadavers in teaching of human anatomy in brazilian medical facuties. Acta Scientiarum Biological Sciences, 2017; 39(1): 1.

5. MEDEIROS ARC, et al. Dissecação e capacitação de habilidades e competências gerais na formação médica. Revista Brasileira de Ciências da Saúde, 2013; 13(3): 247-252.

6. NOBESCHI L, et al. Avaliação sistemática da dissecação como método de ensino e aprendizagem em anatomia humana. Revista Eletrônica Pesquiseduca, 2018; 10(21): 420-432.

7. ÖZCAN S, et al. Impact of cadaveric surgical anatomy training on urology residents knowledge: a preliminary study. Turkish Journal of Urology 2015; 41(2): 83-7.

8. PATEL $\mathrm{K}$, et al. The relationships between learning outcomes and methods of teaching anatomy as perceived by professional anatomists. Clinical Anatomy, 2008; 21: 182-189.

9. PONTINHA CM, SOEIRO C. A dissecação como ferramenta pedagógica no ensino de anatomia em Portugal. Interface, 2014; 18(48): 165-176.

10. RAMOS RFM, et al. Curso básico em cirurgia plástica para acadêmicos: enxertos e retalhos. Revista Amrigs, 2016; 60(4): 279-399.

11. SHEIKH AH, et al. Cadaveric anatomy in the future: What is the surgeos view. Anatomical Sciences Education, 2016; 9: 203-208.

12. SINGH R, et al. Is the decline of human anatomy hazardous to medical education/profession?-A review. Surgical and Radiologic Anatomy, 2015; 37(10): 1257-1265.

13. VAN WYK J, RENNIE CO. Learning anatomy through dissection: Perceptions of a diverse medical student cohort. International Journal of Morphology, 2015; 33(1): 89-95. 